\title{
Experimental evidence for the key role of the ion heat channel in the physics of the L-H transition
}

\author{
F. Ryter, L. Barrera Orte, B. Kurzan, R.M. McDermott, G. Tardini, \\ E. Viezzer, M. Bernert, R. Fischer and the ASDEX Upgrade Team \\ Max-Planck-Institut für Plasmaphysik, D-85748 Garching, Germany
}

\begin{abstract}
.
Experimental investigations carried out in the ASDEX Upgrade tokamak under various conditions demonstrate that the ion heat flux at the plasma edge plays a key role in the L-H transition physics, while the electron heat flux does not seem to play any role. This is due to the fact that the ion heat flux governs the radial electric field well induced by the main ions which is responsible for the turbulence stabilization causing the $\mathrm{L}-\mathrm{H}$ transition. The experiments have been carried out in the low density branch of the power threshold where the electron and ion heat channels can be well separated. In plasmas heated by electron heating, the edge ion heat flux has been increased to reach the L-H transition by using separately three actuators: heating power, density and plasma current. In addition, the key role of the edge ion heating has been confirmed in experiments taking advantage of the direct ion heating provided by neutral beam injection. The role of the ion heat flux explains the non-monotonic density dependence of the L-H threshold power. Based on these results, a formula for the density of the threshold minimum has been developed, which also describes well the values found in tokamaks of various size. For ITER it predicts a value which is close to the density presently foreseen to enter the H-mode and indicates that operation at half field and current would benefit from a very significantly lower density minimum and correspondingly low threshold power.
\end{abstract}

PACS: 52.55.-s 52.55.Dy 52.35.Ra 52.25.Fi

\section{Introduction}

The transition from the low to the high confinement mode in fusion plasmas, named L-H transition, occurs in general above a certain threshold in heating power, labelled $P_{L-H}$. At the L-H transition an edge transport barrier for heat and particles, caused by the reduction of turbulence-driven transport, is formed just inside the last closed magnetic surface. Presently, 
the most favored paradigm to explain this phenomenon is based on turbulence reduction induced by sheared $E \times B$ flow, [1]. In the narrow region at the very edge of the plasma, where the L-H transition occurs, the $E_{r}$ profile exhibits a clear well such that $\nabla E_{r}$ induces a perpendicular sheared flow which stabilizes the turbulence. This hypothesis has been confirmed experimentally for spontaneous L-H transitions, originally in DIII-D, [2], later in most of the devices, see e.g. reviews $[3,4,5]$. The $E_{r}$ well is weakly pronounced in L-mode, much deeper in H-modes, while it takes intermediate values just before the L-H transition, $[4,6]$. It has been shown that the edge $E_{r}$ is essentially induced by the main ions according to neoclassical theory, [7], and therefore mainly determined by the gradient of the main ion pressure, $E_{r}=\nabla p_{i} /\left(e \cdot n_{i}\right)$, where $n_{i}$ is the ion density and $e$ the elementary charge, $[8,5,9]$ and references therein.

In general the transition to H-mode is achieved by increasing the heating power, whereby the plasma density does not change before the L-H transition but $\left|\nabla T_{i}\right|$ increases. This increases $\left|\nabla p_{i}\right|$ and enhances the $E_{r}$ well which becomes more negative and this eventually causes the characteristic turbulence reduction of the transition to $\mathrm{H}$-mode. According to these considerations one expects the ion heat flux at the plasma edge, $q_{i, e d g e}$, to play a key role in the determination of $E_{r}$. In this work, we provide experimental evidence that this is indeed the case, while the electron heat flux does not seem to play any role.

The increase of the heating power on the path to the L-H transition also enhances the turbulence level which excites geodesic acoustic modes and zonal flows leading to selfinduced transient reduction of turbulence and transport through the predator-prey mechanism. This effect, predicted by theory and proposed as a possible triggering mechanism of the L-H transition, [10], has indeed been observed experimentally in several fusion devices prior to the L-H transition, reflected by a modulation of the edge $E_{r}$ profile and turbulence level in typical limit cycle oscillations, also named "IM-mode" or "I-phase", [11, 12, 13, 14, 15, 16].

The density dependence of the L-H power threshold has been known for a long time to be non-monotonic, with a minimum at a given density, labelled here $n_{e, \min }$, [17] and references therein. The power threshold increases on each side of this minimum, in the so-called low and high density branches. The values of $n_{e, \min }$ in the different tokamaks cover a wide range between $2 \times 10^{19} \mathrm{~m}^{-3}$ and $15 \times 10^{19} \mathrm{~m}^{-3},[18]$, whereby the large devices such as JET and JT-60U populate the lower range, the medium size tokamaks DIII-D and ASDEX Upgrade lie somewhat higher, while the highest values are reached in the high field compact Alcator 
C-Mod tokamak. Although some of the variations of $n_{e, \min }$ seem to be related to the magnetic field, this is not sufficient to explain the variation between the devices, [18]. Recent results on this topic have been reported for ASDEX Upgrade in [19, 20], Alcator C-Mod in [21] and JET in [22]. It should be underlined that the threshold scaling expressions yielded by the analysis of the ITPA threshold database are deduced for each device from a data subset in which only the high density branch is considered, $[17,23,18]$. The latest version of the threshold scaling, reads for deuterium plasmas, in MW, [18]:

$$
P_{\text {scal }}=0.049 \bar{n}_{e}{ }^{0.72} B_{T}^{0.80} S^{0.94}
$$

where $\bar{n}_{e}$ is the line-averaged density in $10^{20} \mathrm{~m}^{-3}, B_{T}$ the magnetic field in $\mathrm{T}$, and $S$ the plasma surface area in $\mathrm{m}^{2}$.

The increase of $P_{L-H}$ in the low density branch, i.e. the upwards deviation from the $\bar{n}_{e}{ }^{0.72}$ dependence of the scaling, is observed in most of the tokamaks, but with variable strength $[24,25,26,19,21,20,22]$. The effect is particularly strong in plasmas heated with electron cyclotron resonant heating $(\mathrm{ECRH}),[24,19]$, suggesting that this might be due to the decoupling between the electron and ion heat channels, but this has, so far, not been demonstrated experimentally. We show in this paper that this is indeed the reason as the edge ion heat flux determines the $\mathrm{L}-\mathrm{H}$ transition and, furthermore, that this also explains quantitatively the existence of $n_{e, \min }$ and the large differences found between the tokamaks for this quantity.

The analysis of $q_{i, e d g e}$ requires a separation between the electron and ion heat channels up to the plasma edge from which it is well-known that it can only be achieved if the collisional energy exchange between electron and ion heat channels, $p_{e i}$, is low enough. In practice, this analysis can only be carried out reliably in the low density branch, as done in the present work.

In the remaining of this paper we describe the experimental conditions and the analysis methods in section 2. Section 3 is dedicated to the results on the role of the ion heat flux in the L-H and H-L transitions induced with ECRH, while the analysis of the L-H transitions obtained with neutral beam injection is presented in section 4 . We discuss the physics mechanism which determines $n_{e, \min }$ in section 5 where we also present a comparison with other tokamaks, as well as predictions for ITER. The last section is dedicated to the conclusions. 


\section{Experimental setup and analysis methods}

The experiments have been performed in ASDEX Upgrade, a divertor tokamak with major and minor radii $\mathrm{R}=1.65 \mathrm{~m}$ and $\mathrm{a}=0.5 \mathrm{~m}$ respectively and with an elongation of about 1.6 . All the data are taken from deuterium plasmas in the standard lower single null magnetic configuration with the ion $\nabla B$ drift directed towards the active $\mathrm{X}$-point providing the usual low L-H transition power threshold. ASDEX Upgrade is equipped with all the usual diagnostics, but essential here are the measurements of the electron temperature, $T_{e}$, by the electron cyclotron emission (ECE) and Thomson scattering, as well as the charge exchange recombination spectroscopy (CXRS) which yields the ion temperature, $T_{i}$, and the toroidal plasma rotation, $\mathrm{v}_{\text {tor }}$. An upgrade of the CXRS systems, with a time resolution of $4 \mathrm{~ms}$ in the core and $2 \mathrm{~ms}$ at the plasma edge, enables us since 2011 to also measure these quantities reliably in ECRH heated plasmas using short NBI blips, [27, 28]. The measurement of $T_{i}$ is a fundamental requirement for the analysis of the heat fluxes which can only be carried for discharges from the 2011 campaign and later.

The threshold power, $P_{L-H}$, is obtained, as usual, from $P_{\text {loss }}=P_{\text {heat }}-d W / d t$ at the $\mathrm{L}-\mathrm{H}$ transition, where $P_{\text {heat }}$ is the sum of all the heating powers, W the plasma energy. The $P_{L-H}$ values are obtained from a large number of discharges in which the magnetic field varied between $2.3 \mathrm{~T}$ and $2.5 \mathrm{~T}$. To compensate for these small variations in $B_{T}, P_{L-H}$ is normalized to $2.35 \mathrm{~T}$ by the $B_{T}^{0.8}$ dependence of the threshold scaling, see [20] for details. The ion and electron heat fluxes are provided by time-dependent power balance analyses using the TRANSP code, [29]. Their surface integrated edge values, $Q_{i, e d g e}$ and $Q_{e, \text { edge }}$, are taken at $\rho_{\text {tor }}=0.95$, where $\rho_{\text {tor }}$ is the usual normalized toroidal flux radius. These quantities are in MW which provides a direct comparison with $P_{L-H}$. As usual in power balance analyses, the electron heat flux is corrected for the radiation losses. In ASDEX Upgrade, the electron and ion heat fluxes at the edge can be separated, at the L-H transition, for densities below roughly $4 \times 10^{19} \mathrm{~m}^{-3}$, therefore essentially in the low density branch of the power threshold on which we focus in the present work. However, the non-monotonic density behaviour of $P_{L-H}$, described in detail in reference [20], has been deduced from a wider density range, which will be also used in section 5 of the present paper.

On the basis of nine ECRH-induced L-H transitions very preliminary indications on the importance of $Q_{i, e d g e}$ in the L-H transition physics were reported in [20]. For the present work, the analysis of $Q_{i, e d g e}$ has been extended to about $25 \mathrm{~L}-\mathrm{H}$ transitions covering a wider 
range of parameters, induced with ECRH, as well as with neutral beam injection (NBI) alone or with NBI+ECRH. Moreover, $13 \mathrm{H}-\mathrm{L}$ back transitions could also be analyzed. The value of $B_{T}$ was close to our reference value of $2.35 \mathrm{~T}$ in the discharges for which we could analyze the heat fluxes, such that no $B_{T}^{0.8}$ correction is made.

As mentioned above, in particular at low density, the L-H transitions are often preceded by an I-phase, [14], which evolves gradually towards the H-mode and the time of the transition from I-phase to H-mode cannot always be identified with accuracy. We selected the time points which seemed to be most relevant for the L-H transition.

\section{Investigations with ECRH only}

\subsection{L-H transitions}

The results of our analyses for L-H transitions induced by ECRH only, are illustrated in figure 1 (a) where $P_{L-H}$ and $Q_{i, e d g e}^{L-H}$ are plotted versus the line-averaged density, $\bar{n}_{e}$. As known for the low density branch, $P_{L-H}$ clearly decreases with increasing density. This effect is strong for $1 \mathrm{MA}$ and weaker at $0.6 \mathrm{MA}$, in addition $P_{L-H}$ itself is much lower at $0.6 \mathrm{MA}$ than at $1 \mathrm{MA}$, confirmed here by the addition of several data points at $0.6 \mathrm{MA}$, as compared to reference [20]. Note that the $P_{L-H}$ values for the different currents converge the same value in the high density branch, [20].

The behaviour of $Q_{i, e d g e}^{L-H}$ is fundamentally different from that of $P_{L-H}: Q_{i, \text { edge }}^{L-H}$ increases with increasing density, the points at $1 \mathrm{MA}$ and 0.6 MA exhibit exactly the same values and dependence. This alignment is remarkable considering the large difference in $P_{L-H}$ for the two plasma current values. A linear least square fit of $Q_{i, e d g e}^{L-H}$, not forced through the origin, yields the line drawn in the figure which corresponds to

$$
Q_{i, e d g e}^{L-H, f i t}=0.18 \bar{n}_{e}
$$

in MW and $10^{19} \mathrm{~m}^{-3}$. The values of $Q_{i, e d g e}^{L-H}$ are of course lower than $P_{L-H}$, but this difference can be extremely large, for instance about one order of magnitude at $\bar{n}_{e} \approx$ $1.5 \times 10^{19} \mathrm{~m}^{-3}$. As will be shown below, this is due to the weak collisional energy transfer from the electron to the ion heat channel.

The values of the electron edge heat flux, $Q_{e, e d g e}^{L-H}$, corresponding to the $Q_{i, e d g e}^{L-H}$ data are plotted in figure 1 (b) where the fits to $P_{L-H}$ from panel (a) are also drawn for reference. They are of course lower than $P_{L-H}$ (we remind here that $P_{\text {rad }}$ is subtracted) but exhibit very similar 


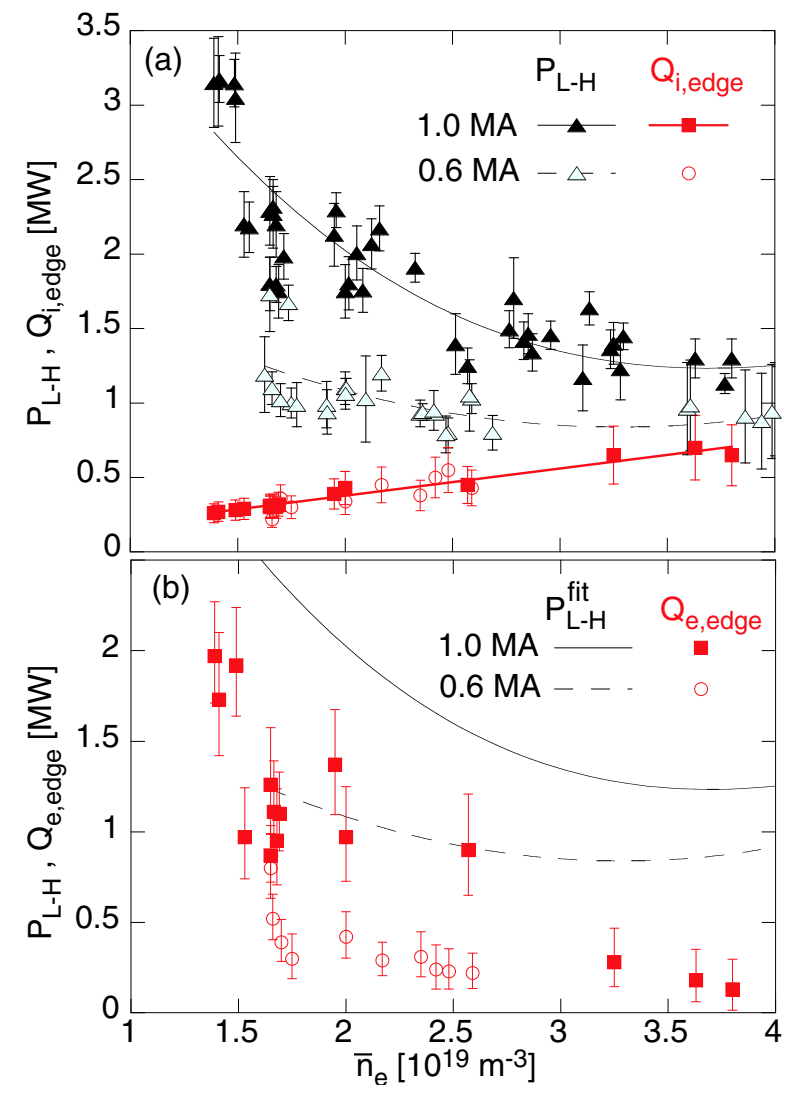

Figure 1. L-H transitions with ECRH: Panel (a): $P_{L-H}$, normalized with $B_{T}^{0.8}$ to $B_{T}=2.35 T$, and $Q_{i, e d g e}^{L-H}$ versus line-averaged density at the $L-H$ transition for $1 M A$ and 0.6 MA discharges. The lines are fits to the data. Panel (b): $Q_{e, e d g e}$ corresponding to the $Q_{i, \text { edge }}$ data from panel $(a)$. The lines are the $P_{L-H}$ fits of panel (a), plotted for reference.

trends. For both current values $Q_{e, e d g e}^{L-H}$ decreases with increasing density which is opposite to the density dependence of $Q_{i, e d g e}^{L-H}$. At $1 \mathrm{MA} Q_{e, \text { edge }}^{L-H}$ is much higher than at $0.6 \mathrm{MA}$ and much larger than $Q_{i, e d g e}^{L-H}$, while these two quantities are closer to each other at 0.6 MA. Therefore, in contrast to $Q_{i, e d g e}^{L-H}, Q_{e, e d g e}^{L-H}$ does not seem to unify the data. It should be pointed out that, as shown in [6] for experiments carried out under similar conditions as those investigated here, the edge temperatures, labelled $T_{j, p e d}$, exhibit very different density dependences: in the density range considered here, $T_{e, p e d}$ decreases by a factor of about 3 as the density is increased, while $T_{i, p e d}$ decreases only by about $35 \%$. Therefore $T_{e, p e d}$, which follows the large variations of $Q_{e, e d g e}^{L-H}$, cannot be considered as a constant critical temperature which would determine the L-H transition. 


\section{2. $H$-L transitions}

Identifying the parameters which determine the H-L back transition contributes to the physics understanding of the transition and is important for predicting ITER which might operate just above the power threshold. The results for our H-L transitions are shown in figure 2 (a) where, similarly to the previous figure, $P_{H-L}$ and $Q_{i, e d g e}^{H-L}$ are plotted. For comparison with the L-H data of figure 1 , we included the fits of $P_{L-H}$ at $1 \mathrm{MA}$ and $0.6 \mathrm{MA}$, as well as $Q_{i, e d g e}^{L-H, f i t}$. As discussed in detail in reference [20], the threshold power at the $\mathrm{H}-\mathrm{L}$ transition, $P_{H-L}$, exhibits a non-monotonic density dependence which is very similar to that of $P_{L-H}$. In addition, there is also the same large difference between $1 \mathrm{MA}$ and 0.6 MA. As reported in [20], in the low density branch, the $P_{H-L}$ data points are close to those of $P_{L-H}$, or higher due to high radiation losses, as clearly visible for the 1 MA dataset in figure 2.

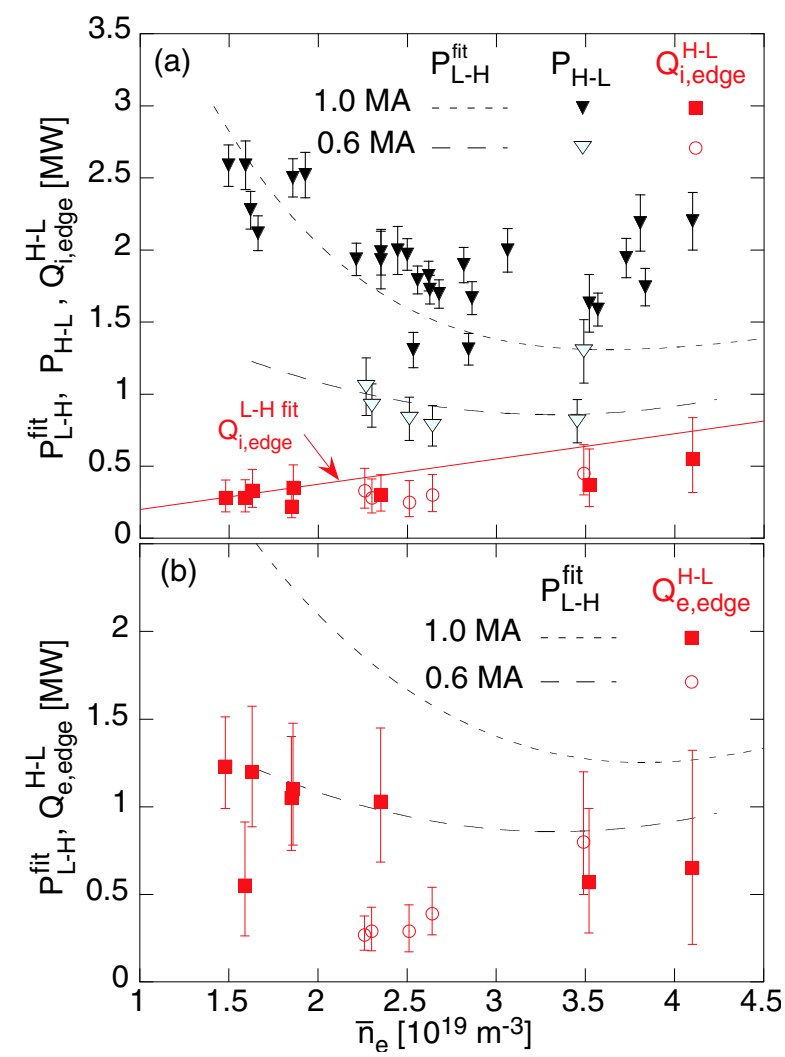

Figure 2. H-L transitions with ECRH: Panel $(a): P_{H-L}$, normalized with $B_{T}^{0.8}$ to $B_{T}=2.35 T$, and $Q_{i, e d g e}^{H-L}$ versus line-averaged density at the $H$ - $L$ transition for $1 M A$ and 0.6 MA discharges. Panel (b): $Q_{e, e d g e}$ corresponding to the $Q_{i, e d g e}$ data from panel (a). In both panels the lines are the $P_{L-H}$ and $Q_{i, e d g e}^{L-H}$ fits from figure 1.

We could also deduce the edge ion and electron heat fluxes for 13 of these H-L transitions 
for which the $T_{i}$ measurement was available and the $d W / d t$ contribution to $P_{\text {loss }}$ not too large. The values of $Q_{i, e d g e}^{H-L}$, reported in figure 2 (a), are close to those for the $\mathrm{L}-\mathrm{H}$ transitions as shown by the comparison with $Q_{i, e d g e}^{L-H}$. It It should be underlined that this is consistent with results on the edge electron pressure in the high density branch at the $\mathrm{L}-\mathrm{H}$ and $\mathrm{H}-\mathrm{L}$ transitions reported in [30]. As for the L-H transitions, for the H-L points the edge ion heat flux values at 1 MA and 0.6 MA are also very close to each other, in contrast to $P_{H-L}$. The H-L transition is experimentally often not well controlled and the uncertainties are significantly larger than for the $\mathrm{L}-\mathrm{H}$ transition due to a larger $\mathrm{dW} / \mathrm{dt}$ contribution. This is particularly true for the time-dependent correction of $Q_{i}$ due to the limited time resolution of the $T_{i}$ measurement determined by the spacing of NBI blips, such that $Q_{i, e d g e}^{H-L}$ might be underestimated. Within the error bars, the edge ion heat flux values at the $\mathrm{L}-\mathrm{H}$ and $\mathrm{H}-\mathrm{L}$ transitions are comparable, but $Q_{i, e d g e}^{H-L}$ is certainly not higher than $Q_{i, e d g e}^{L-H}$. For $\bar{n}_{e}>2.2 \times 10^{19} \mathrm{~m}^{-3}$ the $Q_{i, e d g e}^{H-L}$ values are systemically below the $Q_{i, e d g e}^{L-H, f i t}$ line, such that the present dataset might suggest the possible existence of an hysteresis, defined as $Q_{i, e d g e}^{H-L}<Q_{i, e d g e}^{L-H}$, but does not allow us to assess it yet. The values of $Q_{e, e d g e}^{H-L}$ are plotted in figure 2 (b). As for the $\mathrm{L}-\mathrm{H}$ transitions, $Q_{e, \text { edge }}^{H-L}$ at $1 \mathrm{MA}$ is overall larger than at 0.6 MA. At $1 \mathrm{MA} Q_{e, e d g e}^{H-L}$ decreases with increasing density whereas it seems to exhibit the opposite trend at 0.6 MA. Thus, for the H-L transition also, $Q_{e, e d g e}^{H-L}$ does not seem to provide a coherent physics picture.

\subsection{Ion heat flux in ECRH-heated plasmas}

In this subsection we present experimental analyses which illustrate the dependence of the electron-ion collisional energy transfer upon $T_{e}$ and its impact on $Q_{i, e d g e}$. We show how the results of the previous two subsections can be assessed and explained by the dependence of $Q_{i, e d g e}$ on $T_{e}$.

In ECRH-heated plasmas the input power (ECRH and Ohmic) flows purely into the electron channel. The ion heating occurs exclusively through the electron-ion collisional energy exchange, $p_{e i} \propto n_{e} n_{i}\left(T_{e}-T_{i}\right) T_{e}^{-3 / 2}$, which increases monotonically with density at fixed temperature, but exhibits, at fixed density, a saturation and rollover with increasing $T_{e}$ due to $T_{e}^{-3 / 2}$.

This is demonstrated experimentally with a $1 \mathrm{MA}$ discharge at $\bar{n}_{e}=1.5 \times 10^{19} \mathrm{~m}^{-3}$ in which $P_{E C R H}$ has been increased in steps. Figure 3 (a) illustrates the strong increase of $T_{e}$ with $P_{E C R H}$, while $T_{i}$ varies very little, in agreement with the results reported in [6]. As shown 


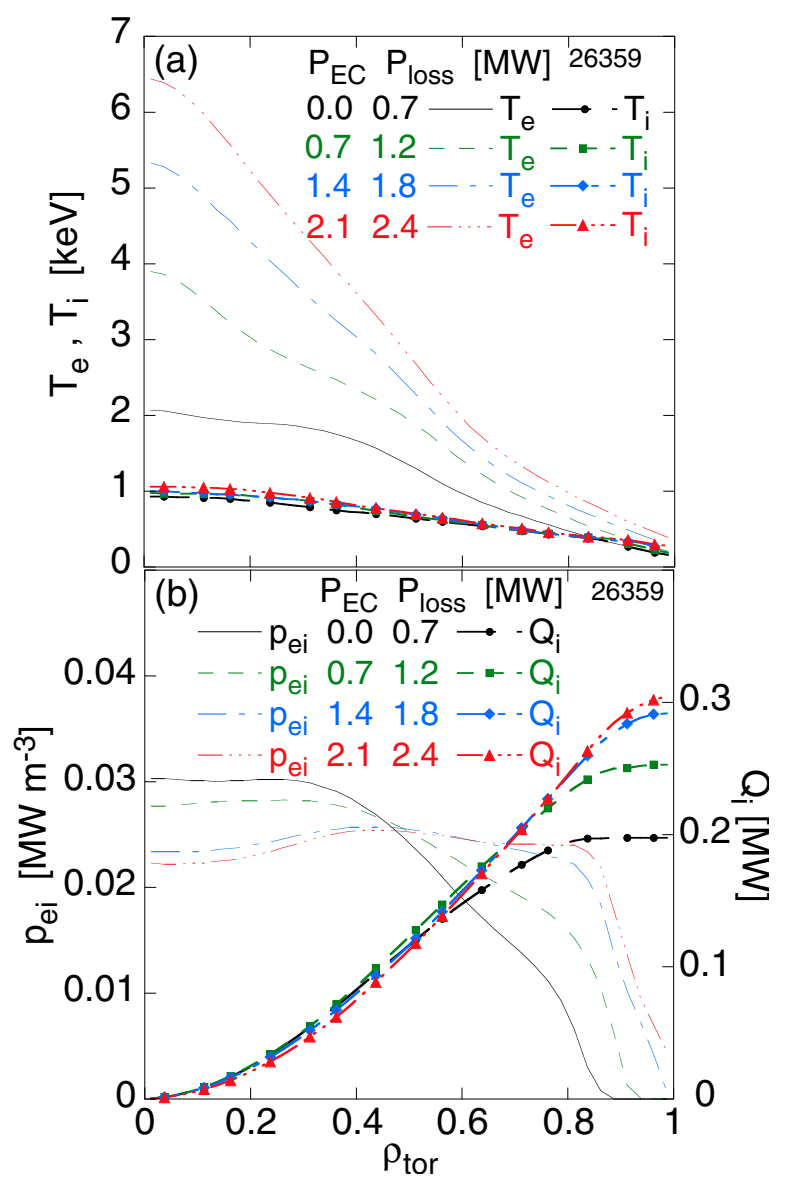

Figure 3. Scan of $P_{E C R H}$ at $I_{p}=1 M A, L$-mode up to L-H transition. Panel (a): $T_{e}$ and $T_{i}$ profiles, $P_{E C}$ and $P_{l o s s}$ are indicated for each case in the legend. Panel (b): $p_{e i}$ and $Q_{i}$ profiles. The symbols are not data points but markers for different curves.

in figure $3(\mathrm{~b})$, in the plasma center $\left(\rho_{t o r}<0.4\right)$, where $T_{e}$ is very high, $p_{e i}$ decreases already for the first step of $P_{E C R H}$ and further with the following steps. The increase of $Q_{i, e d g e}$ arises from the region $\rho_{\text {tor }}>0.5$ where $T_{e}^{-3 / 2}$ does not counter-balance the increase of $T_{e}-T_{i}$. It should be underlined that, while the heating power $P_{l o s s}$ is increased by more than a factor of three $(0.7-2.4 \mathrm{MW}), Q_{i, e d g e}$ increases only from 0.2 to $0.3 \mathrm{MW}$, reflecting the saturation effect. However, in this case, the increase of $Q_{i, e d g e}$ remains monotonic in this range and the L-H transition just occurs during the last ECRH step.

A further contribution to the assessment of the role of the ion heat flux is provided by the analysis of a selection of discharges at $1 \mathrm{MA}$ in a density range $1.25 \times 10^{19}<\bar{n}_{e}<$ $1.7 \times 10^{19} \mathrm{~m}^{-3}$ at different $P_{E C R H}$ values. The $Q_{i, e d g e}$ data in L-mode and at the L-H transition are plotted in figure 4 versus density. All the L-mode points lie below the L-H data confirming 


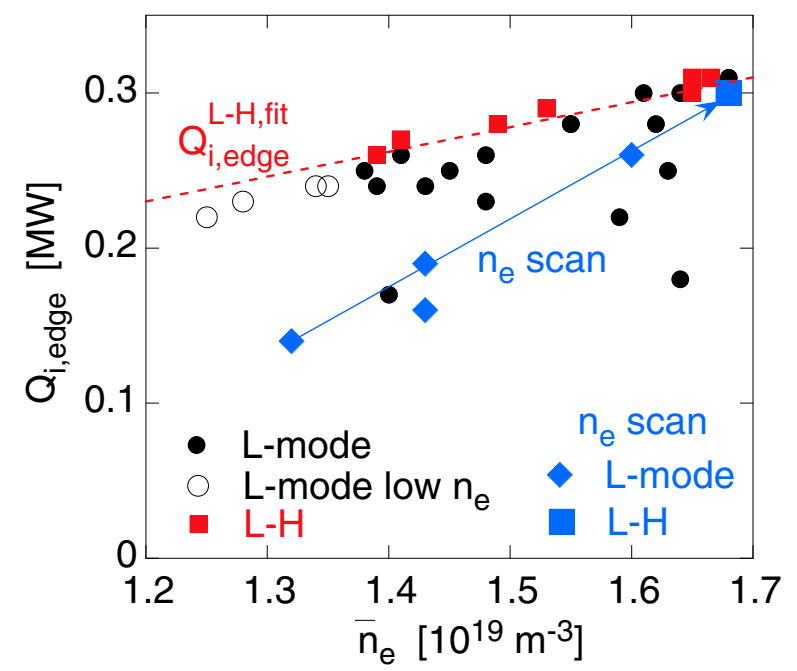

Figure 4. $Q_{i, e d g e} v e r s u s$ density in a limited range for L-mode and L-H points, the dashed line corresponds to $Q_{i, e d g e}^{L-H, f i t}$. The arrow underlines the density scan.

without ambiguity that no L-H transition occurs for values below $Q_{i, e d g e}^{L-H, f i t}$ deduced from figure 1 (a). Also included are the points from a density scan at constant ECRH power for which the L-H transition occurred when the density was sufficiently high, as indicated by the point marked specifically. Its $Q_{i, e d g e}^{L-H}$ value is in perfect agreement with the other L-H points achieved by increasing $P_{E C R H}$, a further confirmation of the key role played by $Q_{i, e d g e}$ which has been increased through $\bar{n}_{e}$ only, at constant heating power.

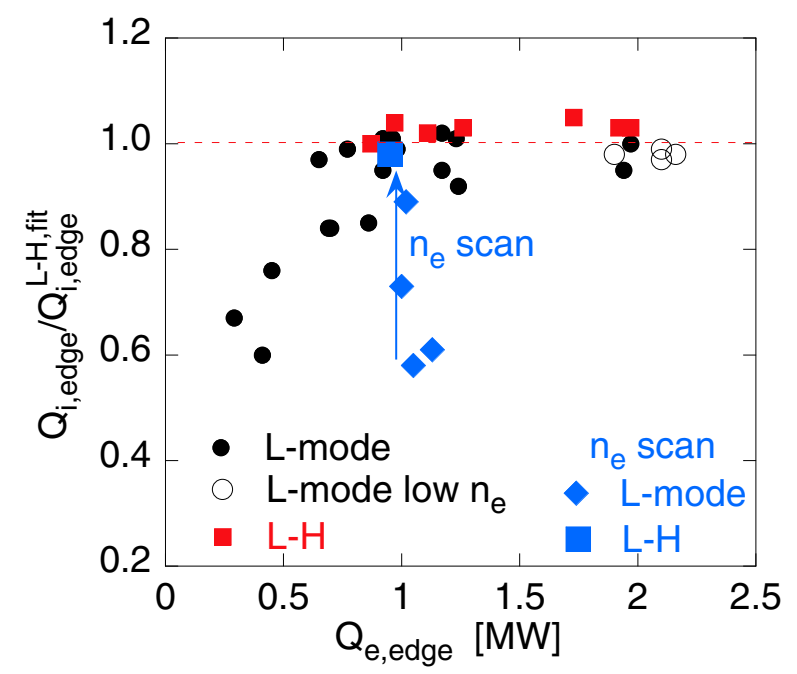

Figure 5. $Q_{i, \text { edge }}$ normalized to $Q_{i, \text { edge }}^{L-H, \text { fit }}$ versus $Q_{e, \text { edge }}$ for the data of figure 4.

The same $Q_{i, e d g e}$ data, normalized to $Q_{i, e d g e}^{L-H, f i t}$ to take the density variation into account, 
are plotted versus $Q_{e, e d g e}$ in figure 5. The L-mode points indicate clearly the increase and saturation of $Q_{i, e d g e}$ with electron heating power. Furthermore, the attention should be drawn to the low density L-mode points (open circles) at the maximum of $Q_{e, e d g e}$ : they are at such a low density (see figure 4 ) that $Q_{i, e d g e}$ reaches the saturation level and rolls over below the value required for the $\mathrm{L}-\mathrm{H}$ transition. This is an experimental confirmation that below a certain density the L-H transition cannot be achieved with pure electron heating because $p_{e i}$ remains too low, independently of $T_{e}$. For completeness the points of the density scan are also reported in figure 5, showing that $Q_{e, e d g e}$ was indeed kept constant.

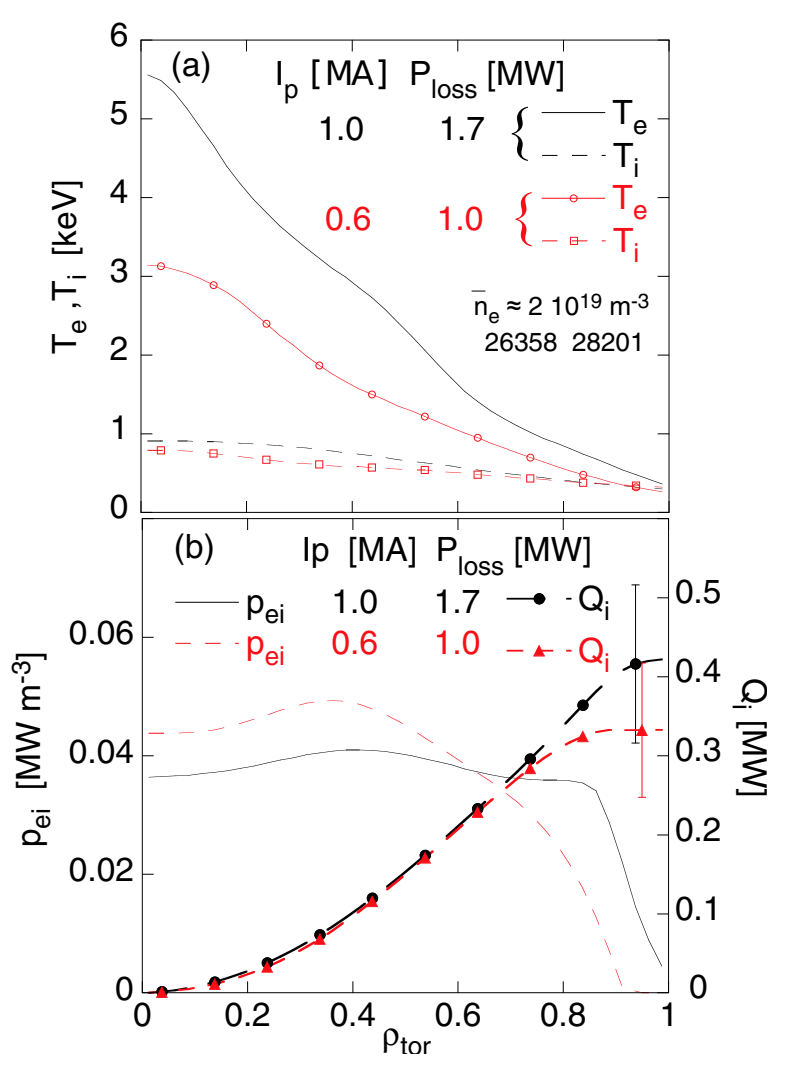

Figure 6. Comparison of data at the L-H transition for two discharges at $1 M A$ and 0.6 MA at the same density. Panel (a): $T_{e}$ and $T_{i}$ profiles, current and $P_{\text {loss }}$ values are indicated in the legend. Panel (b): $p_{e i}$ and $Q_{i}$ profiles. The symbols are not data points but markers for different curves.

The dependence of $p_{e i}$ on $T_{e}$ also explains the same $Q_{i, e d g e}^{L-H}$ values for the $1 \mathrm{MA}$ and 0.6 MA cases. It is well-known that confinement increases with plasma current, such that the temperatures are higher in the $1 \mathrm{MA}$ discharges than at 0.6 MA. As mentioned above, figure 3 shows that in Ohmic plasmas at $1 \mathrm{MA} p_{e i}$ is already close to its saturation and that 
adding ECRH power reduces the central contribution of $p_{e i}$ to the ion heat flux. Due to the low confinement at 0.6 MA, the situation is different: adding ECRH power increases $p_{e i}$ in the center and over a large part of the radius. This is illustrated in figure 6 for data taken just before the L-H transition in two discharges at $1 \mathrm{MA}$ and $0.6 \mathrm{MA}$, both at $\bar{n}_{e}=2 \times 10^{19} \mathrm{~m}^{-3}$. Panel (a) shows the very different $T_{e}$ profiles while $T_{i}$ is comparable. Note the large difference in $P_{\text {loss }}$, as indicated in the legend, which of course corresponds to the values of figure 1. Panel (b) of figure 6 illustrates how the different $p_{e i}$ profiles lead to the same edge ion heat flux, within the error bars. The corresponding $Q_{i, e d g e}^{L-H}$ points are reported in figure 1 (a) and identifiable at $\bar{n}_{e}=2 \times 10^{19} \mathrm{~m}^{-3}$. At $1 \mathrm{MA}$ the high $T_{e}$ in the central plasma reduces $p_{e i}$, while the large $T_{e}-T_{i}$ value in the edge region contributes significantly to $Q_{i, e d g e}$. At $0.6 \mathrm{MA}$ the situation is reversed: the main contribution arises from the core, whereas only the very edge characterized by $T_{e} \approx T_{i}$, does not contribute.

Summarizing this section: The experimental analysis of ECRH-induced L-H transitions in the low density branch strongly suggests that the edge ion heat flux is a key parameter in the L-H transition physics. The main quantities which impact on $Q_{i, e d g e}$ have been varied and yield the same results for the L-H transition. As demonstrated in the last sub-section, the calculation of $Q_{i, e d g e}$ under the experimental conditions used here is accurate, in particular because $T_{e}-T_{i}$ is clearly outside of the experimental error bars over the main part of the plasma radius.

\section{L-H transitions with NBI}

To further assess the importance of the ion heat flux in the L-H transition physics we performed L-H transitions in the low density branch of 1 MA plasmas heated by NBI at a low acceleration voltage of $35 \mathrm{kV}$. Under such conditions, as $T_{e}$ is high, the ion fraction of the NBI heating is larger than $50 \%$ and we expect the L-H transitions to happen at lower $P_{L-H}$ values than with ECRH only. Due to the low density the NBI penetrates deeply into the plasma despite the low acceleration voltage. We applied either NBI alone or combined with ECRH, whereby the ECRH power was turned on before the NBI and then kept constant. This applied $P_{E C R H}$ was at most $0.7 \mathrm{MW}$ which is significantly lower than the required power to trigger an $\mathrm{L}-\mathrm{H}$ transition in this density region, see figure 1. The L-H transitions have been achieved by increasing the NBI in steps which yields data in L-mode at different NBI and ECRH powers, as well as the L-H points. We made a series at $\bar{n}_{e} \approx 1.75 \times 10^{19} \mathrm{~m}^{-3}$, extended by two L-H points at higher density, $\bar{n}_{e} \approx 2.2 \times 10^{19} \mathrm{~m}^{-3}$ and $\bar{n}_{e} \approx 2.6 \times 10^{19} \mathrm{~m}^{-3}$. The results are shown 
in figure 7 where $P_{L-H}, Q_{i, e d g e}^{L-H}$ and $Q_{e, e d g e}^{L-H}$ are plotted. As reference the $P_{L-H}$ values of figure 1 for $1 \mathrm{MA}$ with ECRH alone are also reported. The values of $P_{E C R H}$ for the NBI(+EC) cases are indicated in the plot.

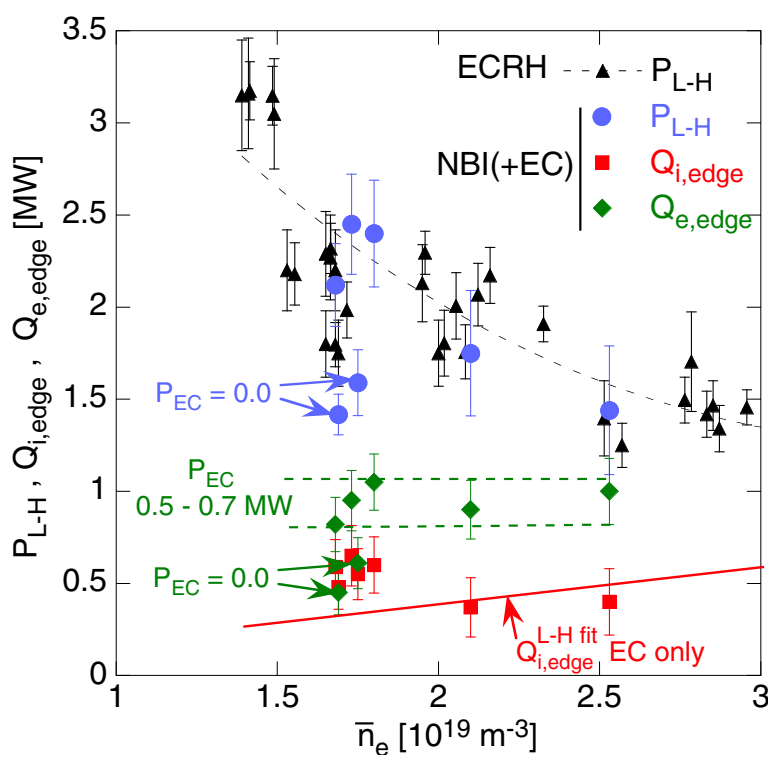

Figure 7. L-H transitions at 1 MA: $P_{L-H}$ for ECRH transitions normalized with $B_{T}^{0.8}$ to $B_{T}=2.35 T$ and corresponding fit from figure 1. $P_{L-H}, Q_{i, e d g e}^{L-H}$ and $Q_{e, e d g e}^{L-H}$ for the $N B I(+E C)$ cases versus line-averaged density. For reference $Q_{i, e d g e}^{L-H, f i t}$ of figure 1 is also reported.

We first focus on the series at $\bar{n}_{e} \approx 1.75 \times 10^{19} \mathrm{~m}^{-3}$. With NBI alone (solid circles labelled $\left.P_{E C}=0\right) P_{L-H}$ is about $40 \%$ lower than the ECRH points at the same density. This agrees with the expectation and demonstrates the importance of ion heating in the L$\mathrm{H}$ transition, as discussed in more detail in the following. If $P_{E C R H}$ is combined with NBI, $P_{L-H}$ increases by at least the amount of $P_{E C R H}$ and reaches the level of the L-H points with ECRH only. The fact that the electron heating indeed increases is shown by $Q_{e, e d g e}^{L-H}$, diamonds in the figure. Thus, at constant density, both $P_{L-H}$ and $Q_{e, e d g e}^{L-H}$ vary with $P_{E C R H}$. In contrast, the corresponding $Q_{i, e d g e}^{L-H}$ points (at $\bar{n}_{e} \approx 1.75 \times 10^{19} \mathrm{~m}^{-3}$ ) exhibit all the same value, independently of $P_{E C}$, showing that the added ECRH power does not contribute to the triggering of the L-H transition. This is due to the fact that the ion heat flux is dominated by the NBI contribution. In fact, the measurements indicate that, before the $\mathrm{L}-\mathrm{H}$ transition, $T_{i}$ is clearly larger than $T_{e}$ over the whole radius. As a consequence of this situation, the impact of $P_{E C R H}$ on $Q_{i, e d g e}$ through $T_{e}$ and $p_{e i}$ is negligible. In summary, these results with NBI yield 
a further evidence for the main role played by the ion heat flux in the L-H transition physics. However, it should be underlined that the $Q_{i, e d g e}^{L-H}$ values with NBI at $\bar{n}_{e} \approx 1.75 \times 10^{19} \mathrm{~m}^{-3}$ lie significantly above the values induced by ECRH only, represented by $Q_{i, e d g e}^{L-H, f i t}$ in figure 7 , while the two points with NBI at higher density agree with this fit.

The higher values of $Q_{i, \text { edge }}^{L-H}$ for the $\mathrm{NBI}(+\mathrm{EC})$ cases at $\bar{n}_{e} \approx 1.75 \times 10^{19} \mathrm{~m}^{-3}$ are explained by the effect of plasma rotation as follows. Power threshold studies in the DIII-D tokamak using NBI torque variation with co-current and counter-current beams revealed that $P_{L-H}$ increases with increasing toroidal edge plasma rotation, $\mathrm{v}_{\text {tor,edge }}$, [31].

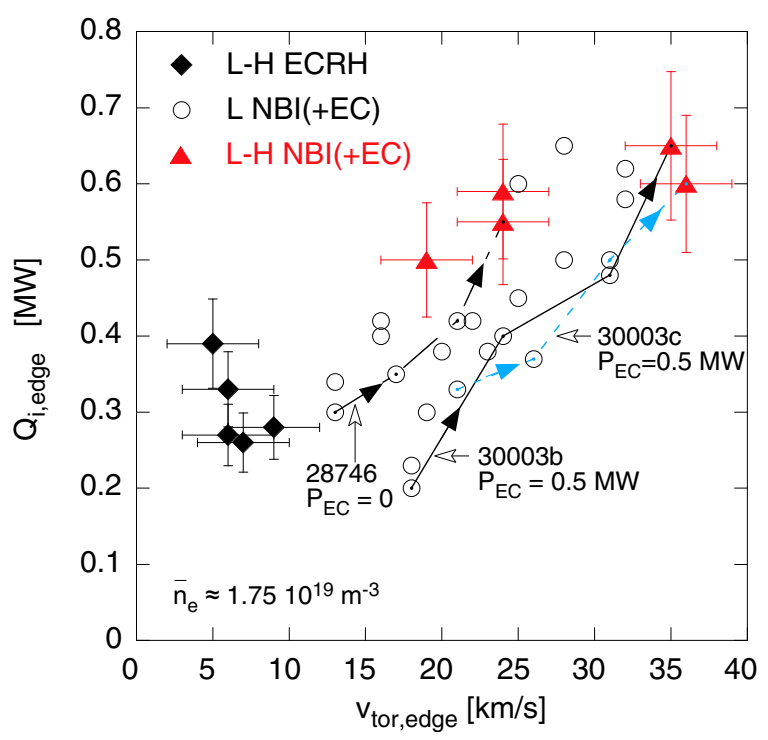

Figure 8. $Q_{i, \text { edge }}$ versus $\mathrm{v}_{\text {tor,edge }}$ for ECRH-induced L-H transitions, as well as L-mode and $L-H$ data obtained with NBI. The trajectories of three scans in NBI, at fixed $P_{E C R H}$, are indicated by the lines.

As mentioned above, in the $\mathrm{NBI}(+\mathrm{EC})$ series at $\bar{n}_{e} \approx 1.75 \times 10^{19} \mathrm{~m}^{-3}$ we could gather Lmode data at different values of NBI power and torque up to the L-H transition. The toroidal rotation at $\rho_{\text {tor }}=0.95$ has been measured with the edge CXRS diagnostic. The quality of the $\mathrm{v}_{\text {tor,edge }}$ measurement is assessed by the fact that, in this narrow density range, the values are consistent with the calculated applied NBI torque yielded by TRANSP, $T_{N B I}$. In fact, at this density we find for $\mathrm{v}_{\text {tor,edge }}$ an off-set linear relation: $\mathrm{v}_{\text {tor,edge }}=8.7+17 \times T_{N B I}$ in $\mathrm{km} / \mathrm{s}$ and $\mathrm{N} \times \mathrm{m}$. The off-set of $8.7 \mathrm{~km} / \mathrm{s}$ without external torque is in agreement with the values reported in [27]. The results for the L-mode and L-H transitions are illustrated in figure 8 by $Q_{i, e d g e}$ plotted versus $\mathrm{v}_{\text {tor,edge }}$ for the $\mathrm{NBI}(+\mathrm{EC})$ scan. For comparison L-H points with ECRH only, at this density, are also reported and exhibit, as expected, the lowest edge 
rotation. The L-H data points with NBI show an increase of $Q_{i, e d g e}^{L-H}$ associated with $\mathrm{v}_{\text {tor,edge }}$ such that $Q_{i, e d g e}^{L-H}$ with NBI can be about two times larger than for the ECRH cases at the same density. This doubling of $Q_{i, e d g e}^{L-H}$ for an increase of $\mathrm{v}_{\text {tor,edge }}$ from about $8 \mathrm{~km} / \mathrm{s}$ to $35 \mathrm{~km} / \mathrm{s}$ is in quantitative agreement with the DIII-D results for $P_{L-H}$, also obtained at low density $\left(\bar{n}_{e} \approx 2-2.5 \times 10^{19} \mathrm{~m}^{-3}\right)$, [31]. As the two tokamaks are very similar in shape and size this comparison is valid. As indicated by figure 8, the L-mode points with NBI are all below the boundary suggested by the L-H points, which here also indicates that no L-H transition occurs at lower $Q_{i, e d g e}$ values. This is confirmed by the paths to the $\mathrm{L}-\mathrm{H}$ transition shown for three NBI power scans. They indicate that the occurrence of the L-H transition is the result of a competition between the increase of $Q_{i, e d g e}$ and that of $\mathrm{v}_{\text {tor,edge }}$ as the NBI power is applied: the increase of $\mathrm{v}_{\text {tor,edge }}$ in parallel with $Q_{i, e d g e}$ prevents the L-H transition to occur at lower NBI power. This can be explained qualitatively by the contribution of the parallel flow to the $E_{r}$ profile, see e.g. [5, 32], but a quantitative assessment of this contribution, which requires an accurate measurement of the radial gradient of the toroidal rotation in the very edge, is not possible with the present data.

Taking into account data over a larger density range we found that $\mathrm{v}_{\text {tor,edge }}$ in the L-mode at different external torque values decreases strongly with increasing density, roughly as $\bar{n}_{e}^{-1.7}$. The somewhat higher values of $\mathrm{v}_{\text {tor,edge }}$ visible in figure 8 for the discharges where ECRH was added to NBI (30003b 30003c) are attributed to slight changes of the density profile induced by the electron heating for the lower NBI power steps. The two points at higher density, $2.2 \times 10^{19} \mathrm{~m}^{-3}$ and $2.6 \times 10^{19} \mathrm{~m}^{-3}$, plotted in figure 7 exhibit a low value of $\mathrm{v}_{\text {tor,edge }}$ which explains that they agree with $Q_{i, e d g e}^{L-H, f i t}$.

A possible dependence of $P_{L-H}$ on $\mathrm{v}_{\text {tor,edge }}$ in ASDEX Upgrade has been investigated previously in comparisons between NBI and ECRH induced L-H transitions at densities above $4 \times 10^{19} \mathrm{~m}^{-3}$ and no difference was found within the accuracy of $P_{L-H}$, [19]. This agrees with the rather low edge toroidal rotation $\mathrm{v}_{\text {tor,edge }} \approx 15 \mathrm{~km} / \mathrm{s}$ measured at the NBI-induced L-H transition under such conditions which is due to the low $P_{L-H}(1.5 \mathrm{MW})$ and correspondingly low torque, combined with the higher density. Therefore, the results reported in the present paper are not in contradiction with our previous work. 


\section{Determination of $n_{e, \min }$}

As suggested by the results presented in the previous sections, the minimum threshold density seems to be determined by the collisional coupling between the electron and ion heat channels, such that below a given density $p_{e i}$ is not large enough to fully equilibrate the electron and ions temperatures and fluxes, even in the edge region: the edge ion heat flux depends on the core input powers in the respective channels. Further, it should be underlined that the decrease of $P_{L-H}$ with decreasing density in the high density branch leads to an increase of the relative contribution of the Ohmic power to the total heating power and therefore an increase of the electron heating, even if the auxiliary heating provides some ion heating. If this electron heating fraction is not fully equilibrated by the exchange term at the plasma edge, it is reflected in a higher $P_{L-H}$ value.

The collisional electron-ion energy transfer is well-known to depend on the ratio $\tau_{E} / \tau_{e i}$ where $\tau_{E}$ is the energy confinement time and $\tau_{e i}$ the volume-averaged electron-ion energy exchange time. Figure 9, which shows $P_{L-H}$ plotted versus $\tau_{E} / \tau_{e i}$ at $1 \mathrm{MA}$ and 0.6 MA, indicates that for both values of the plasma current the minimum of $P_{L-H}$ occurs at $\tau_{E} / \tau_{e i} \approx 9$. Here the whole density range of our threshold studies, up to $7 \times 10^{19} \mathrm{~m}^{-3}$ as shown in [20] figure 3 , has been included.

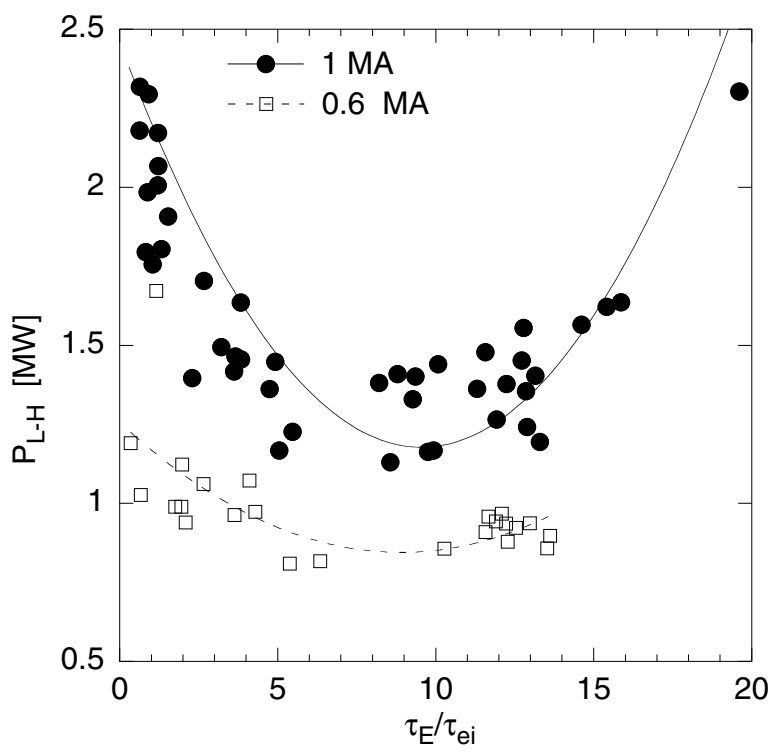

Figure 9. Experimental value of $P_{L-H}$ versus $\tau_{E} / \tau_{e i}$ at $1 \mathrm{MA}$ and $0.6 \mathrm{MA}$. The lines are quadratic fits to the respective data.

Based on this result, a general formula for $n_{e, \min }$ can be derived by combining the the 
threshold scaling, Eq. 1, and the L-mode confinement scaling, Eq. 7 in [33]. For this purpose $\tau_{E}$ and $\tau_{e i}$ at the L-H transition are calculated, whereby the heating power is provided by the threshold scaling and $n_{e, \min }$ is determined by $\tau_{E} / \tau_{e i}=9$. This yields, for deuterium plasmas, the following expression:

$$
n_{e, \text { min }}^{\text {scal }} \approx 0.7 I_{p}^{0.34} B_{T}^{0.62} a^{-0.95}(R / a)^{0.4}
$$

in $10^{19} \mathrm{~m}^{-3}$ using MA, T and $\mathrm{m}$. This formula exhibits an increase with $I_{p}$ and $B_{T}$, but a decrease with machine size due to the fact that $\tau_{E}$ increases strongly with increasing machine size. It is worth noticing that the $I_{p}$ dependence is in agreement with the variation of $n_{e, \min }$ yielded by our data.

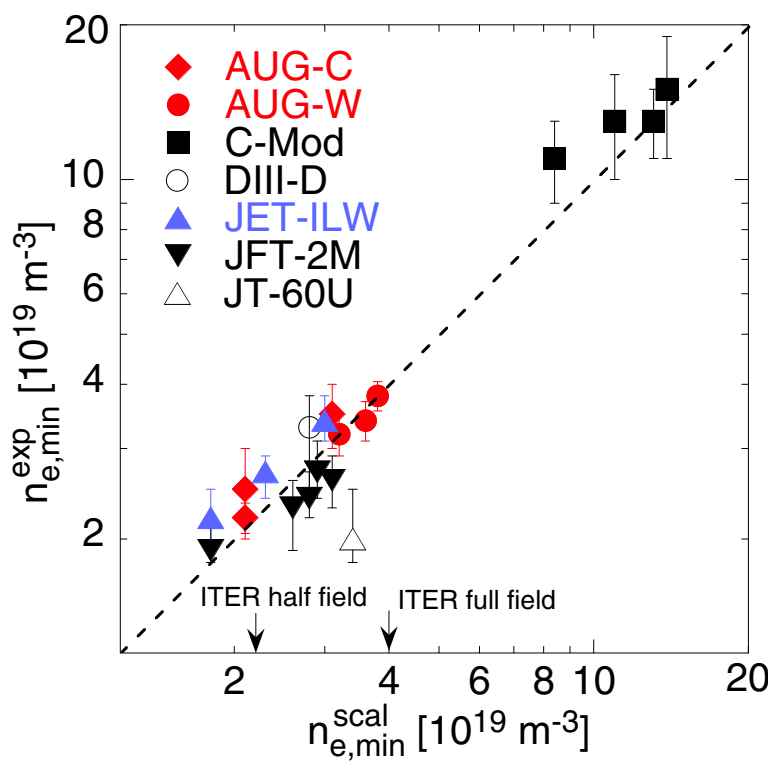

Figure 10. Experimental value of $n_{e, \min }$ versus $n_{\text {e.min }}^{\text {scal }}$ prediction from equation 3. AUG$C$ stands for carbon wall, AUG-W for tungsten wall, JET-ILW for beryllium/tungsten ITER-Like Wall. The dashed line represents $n_{e, \min }^{\text {exp }}=n_{e . \min }^{\text {scal }}$. Data references: $C$-Mod [21], JET-ILW [22], DIII-D, JFT-2M and JT-60U [18].

This formula can be applied to data from various devices: the recent results from Alcator C-Mod in [21] and JET with the ITER-Like Wall in [22], as well as those deduced from the ITPA threshold database and reported in [18] for DIII-D, JFT-2M and JT-60U. The comparison between the experimental and predicted values of $n_{e, \min }$ is illustrated in figure 10. The experimental $n_{e, \min }$ values in all the devices are well reproduced by this formula, in particular the high values found in Alcator C-Mod compared to the other tokamaks, which result from high $I_{p}$ and $B_{T}$ values combined with the small machine size. The value predicted 
by the formula for JT-60U is clearly too high which cannot be explained by the larger aspect ratio which only contributes by $15 \%$ compared to JET. This is further supported by the good agreement for the JFT-2M data, despite the fact that the aspect ratio of this device is larger than that of JT-60U. We also underline that, for ASDEX Upgrade, the difference between the carbon and tungsten walls is small. It is worth noticing that the $B_{T}^{4 / 5}$ of $n_{e, \text { min }}$ reported for JET in [22] is in good agreement with equation 3, explained by the fact that in these JET experiments $I_{p}$ was varied almost proportionally to $B_{T}$. Our formula also explains that Ohmic H-modes could be achieved in ASDEX Upgrade with $B_{T}=1.1 \mathrm{~T}$ at densities as low as $n_{e, \text { min }} \approx 2.2 \times 10^{19} \mathrm{~m}^{-3}$ : the low $B_{T}$ value does not only yield a low $P_{L-H}$ through $B_{T}^{0.8}$, but also reduces $n_{e, \min }$ such that the L-H transition is achieved by reducing the density, as reported in [34].

If NBI heating is used in ITER, the lowest density is determined by the maximum tolerable heat load on the inner wall caused by the shine-through. An recent improvement of the inner wall component design and an new assessment of the maximum tolerable power load by the NBI shine-through yield a minimum density determined by the NBI shine-through in the range $2.5-3 \times 10^{19} \mathrm{~m}^{-3}$, depending on the experimental conditions and gas species, [35]. The previous assumptions and design yielded $5 \times 10^{19} \mathrm{~m}^{-3}$. Equation 3 predicts $n_{e, \text { min }} \approx 4 \times 10^{19} \mathrm{~m}^{-3}$ in ITER at full field and current, which is a bit higher than the minimum tolerable density. At half field and current, a potential scenario for the non-nuclear phase, equation 3 yields $n_{e, \min } \approx 2.2 \times 10^{19} \mathrm{~m}^{-3}$, somewhat lower than the limit. Overall, considering the uncertainties of the extrapolation of $n_{e, \min }$, the present design of ITER seems to allow the $\mathrm{H}$-mode access very close to the minimum threshold power.

Inserting the expression for $n_{e, \min }$ in the threshold scaling, yields a relationship for the power threshold minimum:

$$
P_{L-H}^{\min }=0.36 I_{p}^{0.27} B_{T}^{1.25} R^{1.23}(R / a)^{0.08}
$$

in MW using MA, T and $\mathrm{m}$ for deuterium plasmas. This yields for ITER deuterium plasmas at full field $P_{L-H}^{\min } \approx 41 \mathrm{MW}$, which is somewhat lower than the $52 \mathrm{MW}$ calculated for previous limit of $\bar{n}_{e} \approx 5 \times 10^{19} \mathrm{~m}^{-3}$, [18]. At half field and current the formula predicts $P_{L-H}^{\min } \approx 16 \mathrm{MW}$ (at $n_{e, \text { min }} \approx 2.2 \times 10^{19} \mathrm{~m}^{-3}$ ) which seems suitable to access the H-mode even with ECRH only. Note that in a DT mixture these threshold values are expected to be lower by about $20 \%$, following the $1 / M$ mass scaling of the power threshold, [36, 18]. It should be underlined that if the reduction of $P_{L-H}$ induced by the change from the carbon to the 
metallic wall in ASDEX Upgrade, [20], and JET, [22], can be extrapolated to ITER, the above threshold values for ITER might be reduced. These results on the minimum density might be further assessed by a detailed multi-machine study in the frame of the ITPA topical groups.

\section{Discussion and conclusion}

We have shown that the edge ion heat flux plays a key role in the L-H transition physics using four different experimental ways to vary it. Three of them use pure electron heating with ECRH: i) increasing $P_{E C R H}$ at constant density; ii) increasing density at constant ECRH power; iii) changing confinement and therefore $p_{e i}$ through the plasma current. The fourth method consists in reaching the L-H transition with a significant amount of direct ion heating provided by NBI. The edge ion heat flux data provide a coherent pattern for the L-H points, which, in contrast to the electron heat flux and $P_{L-H}$, unifies the L-H transition features achieved with ECRH and NBI at various densities and different plasma currents. The value of the edge ion heat flux at the L-H transition increases linearly with density in the low density branch of $P_{L-H}$ at constant $B_{T}$ and plasma configuration, while $P_{L-H}$ decreases with increasing density in this branch. This opposite behaviour is explained by the properties of the energy transfer from the electron to the ion channel. The L-H transitions induced by NBI, with significant direct ion heating, are induced in this density range by lower $P_{L-H}$ values, but still exhibit the upwards deviation from the $\bar{n}_{e}^{0.72}$ threshold scaling density dependence. This is due to the non-negligible and unavoidable fraction of electron heating.

It has been reported for ASDEX Upgrade that the L-H transition occurs for the same edge $E_{r}$ profile over a wide range in density, [6]. Combining the two relations $E_{r}=\nabla p_{i} /\left(e \cdot n_{i}\right)$ and $q_{i}=-n_{i} \chi_{i} \nabla T_{i}$, where $\chi_{i}$ is the ion heat diffusivity, one obtains $E_{r}=-q_{i} /\left(\chi_{i} e \cdot n_{i}\right)+T_{i} \nabla n_{i} /(e$. $\left.n_{i}\right)$. This indicates that the required constant $E_{r}$ profile is expected to be obtained for a roughly constant value of $q_{i} / n_{i}$, as the variations of $\chi_{i}$ and $\nabla n_{i} / n_{i}$ are expected to be moderate, while $T_{i}$ at the L-H transition varies very little as shown in [6]. This is therefore in agreement with our experimental finding that $Q_{i, e d g e}^{L-H}$ increases linearly with density.

The analysis of $Q_{i, e d g e}^{L-H}$ cannot be carried out in the high density branch $\left(\bar{n}_{e}>4 \times 10^{19} \mathrm{~m}^{-3}\right)$ where the ion and electron channels cannot be separated with sufficient accuracy. However, it should be underlined that extrapolating the relationship $Q_{i, e d g e}^{L-H, f i t}=0.18 \bar{n}_{e}$ to the high density branch is not in contradiction with $P_{L-H}$ in this density range. For instance, at $\bar{n}_{e}=6 \times 10^{19} \mathrm{~m}^{-3} P_{L-H}$ in ASDEX Upgrade is about $1.5 \mathrm{MW}$, [20], while $Q_{i, \text { edge }}^{L-H}$ at this 
density yields 1.1 MW which is realistic and compatible with the value of $P_{L-H}$.

The fact that the L-H transition is mainly determined by the ion heat flux suggests possible explanations for several features of the threshold studies:

- As reported in [14], at low density, the I-phase exists over a wide range of $P_{E C R H}$. This is due to the weak dependence of $Q_{i, e d g e}^{L-H}$ on $T_{e}$ as illustrated in section 3. Along the same line, long-lasting I-phases which evolve spontaneously towards a clear H-mode, at almost constant power, are often observed at low density. This is explained by a weak density increase induced by the limit cycle oscillations which causes a self-induced increase of $Q_{i, e d g e}$, proportional to $n_{e}^{2}$, up to $Q_{i, e d g e}^{L-H}$.

- It is often argued that the L-H transport barrier being an edge phenomenon, the edge density should be used in the power threshold investigations and not the line-averaged density. The edge ion heat flux is the result of heat transport over the whole radius where $p_{e i}$ can strongly contribute and this effect is better taken into account using the line-averaged density. This has been verified on the present dataset. Of course the edge density must be used for local edge analyses.

- The power threshold studies are generally based on $P_{\text {loss }}$ and it is often pointed out that the radiation power inside the separatrix, $P_{r a d}^{s e p}$, should be subtracted. In general, the radiation losses indeed influence $Q_{i, e d g e}$ but this impact can vary significantly, depending on the experimental conditions. Subtracting $P_{r a d}^{s e p}$ from $P_{L-H}$ does not necessarily improve the coherence of the threshold results, as revealed by attempts on the data presented here.

- It has been shown in reference [6] that in the low density branch a very clear $T_{e}$ pedestal develops in L-mode when the ECRH power is increased. This $T_{e}$ pedestal is almost not affected by the L-H transition: an edge transport barrier for the electron heat flux seems to be established well below the actual $P_{L-H}$ value. In contrast, under these conditions $T_{i}$ remains much lower and develops a pedestal only after the $\mathrm{L}-\mathrm{H}$ transition, the edge density as well. In combination with the results presented here, this observation suggests that, at least at low density with electron heating, the edge transport barrier created at the L-H transition by $Q_{i, e d g e}^{L-H}$ and the induced $E_{r}$ well affects ion-driven turbulence, while a barrier for electron heat transport is established much before.

To conclude, a few words to avoid misunderstanding: We have shown that, at fixed magnetic field and for a given magnetic configuration, the edge ion heat flux at the L-H transition 
increases with density and unifies data obtained at different plasma current which can be cast in the expression $Q_{i, e d g e}^{L-H, f i t}$, equation 2 . However, we do not claim that this expression universally explains all the L-H transition experimental characteristics. In particular, it does not include any $B_{T}$ dependence while we have indications that $Q_{i, e d g e}^{L-H}$ increases with $B_{T}$. We could clearly verify on a few points that in hydrogen, for which the power threshold is about 1.8 times higher than in deuterium, [20], $Q_{i, \text { edge }}^{L-H}$ is correspondingly higher than in deuterium plasmas. Similarly, the higher threshold observed for the ion $\nabla B$ drift direction away from the X-point is linked to higher values of $Q_{i, e d g e}^{L-H}$. This suggests that $P_{L-H}$ and $Q_{i, e d g e}^{L-H}$ are higher because either the $\left|\nabla E_{r}\right|$ value required to reduce the turbulence must be larger than in the cases discussed in the present work, or that a stronger edge ion heat flux is required to produce a given $E_{r}$ well, for instance if a flatter $\nabla p_{i}$ is caused by a higher ion heat transport or different conditions in the boundary plasma. Formulated more generally: our study provides evidence for the key role of the edge ion heat flux in the L-H transition physics mechanism, but its absolute value depends on some of the experimental conditions through their impact on turbulence, $E_{r}$ well and their interaction in the mechanism which reduces transport. Future experiments with an upgraded edge CXRS system are foreseen in ASDEX Upgrade to determine the relation between edge $E_{r}$ and ion heat flux at the L-H transition under these various conditions.

\section{Acknowledgement}

We warmly thank Y. Martin for having provided the values of the plasma current corresponding to the data plotted in reference [18] figure 4. Fruitful discussions with A. Kallenbach are highly appreciated. It is a pleasure to acknowledge the excellent support of the ASDEX Upgrade technical staff.

\section{References}

[1] BIGLARI, H. et al., Phys. Fluids B 2 (1990) 1.

[2] GROEBnER, R. J. et al., Phys. Rev. Lett. 64 (1990) 3015.

[3] BURRELL, K. H., Phys. Plasmas 4 (1997) 1499.

[4] WAGNER, F., Plasma Physics and Controlled Fusion 49 (2007) B1.

[5] STROTH, U. et al., Plasma Physics and Controlled Fusion 53 (2011) 024006.

[6] SAUTER, P. et al., Nuclear Fusion 52 (2012) 012001.

[7] HINTON, F. L. et al., Rev. Mod. Phys. 48 (1976) 239.

[8] MCDERMOTT, R. M. et al., Physics of Plasmas 16 (2009) 056103. 
[9] VIEZZER, E. et al., Nuclear Fusion 53 (2013) 053005.

[10] KIM, E. J. et al., Phys. Rev. Lett. 90 (2003) 185006.

[11] MOYER, R. A. et al., Phys. Rev. Lett. 87 (2001) 135001.

[12] COLCHIN, R. J. et al., Phys. Rev. Lett. 88 (2002) 255002.

[13] PUNZMANN, H. et al., Phys. Rev. Lett. 93 (2004) 125003.

[14] CONWAY, G. D. et al., Phys. Rev. Lett. 106 (2011) 065001.

[15] ESTRADA, T. et al., Phys. Rev. Lett. 107 (2011) 245004.

[16] SCHMITZ, L. et al., Phys. Rev. Lett. 108 (2012) 155002.

[17] RYTER, F. et al., Nuclear Fusion, 36 (1996) 1217.

[18] MARTIN, Y. R. et al., Journal of Physics: Conference Series 123 (2008) 012033.

[19] RYTER, F. et al., Nuclear Fusion 49 (2009) 062003.

[20] RYTER, F. et al., Nuclear Fusion 53 (2013) 113003.

[21] MA, Y. et al., Nuclear Fusion 52 (2012) 023010.

[22] MAGGI, C. et al., Nuclear Fusion 54 (2014) 023007.

[23] RYTER, F. et al., Plasma Physics and Controlled Fusion 44 (2002) A415.

[24] FIELDING, S. J. et al., Plasma Phys. Controlled Fusion 40 (1998) 731.

[25] FUKUDA, T. et al., Plasma Phys. Controlled Fusion 42 (2000) A289.

[26] ANDREW, Y. et al., Plasma Physics and Controlled Fusion 48 (2006) 479.

[27] MCDERMOTT, R. M. et al., Plasma Physics and Controlled Fusion 53 (2011) 124013.

[28] VIEZZER, E. et al., Review of Scientific Instruments 83 (2012) 103501.

[29] PANKIN, A. et al., Computer Phys. Comm. 159 (2004) 157.

[30] WILLENSDORFER, M. et al., Nuclear Fusion 52 (2012) 114026.

[31] GOHIL, P. et al., Nuclear Fusion 50 (2010) 064011.

[32] VIEZZER, E. et al., Nuclear Fusion 54 (2014) 012003.

[33] KAYE, S. et al., Nuclear Fusion 37 (1997) 1303.

[34] RYTER, F. et al., Plasma Phys. Controlled Fusion 36 (1994) A99.

[35] POLEVOI, A. et al., Nuclear Fusion 53 (2013) 123026.

[36] RIGHI, E. et al., Nucl. Fusion 39 (1999) 309 . 